ON THE OBSTACLES TO THE RE-ESTABLISHMENT

or

NATURAL RESPIRATION

AFTER THE

\title{
PERFORMANCE OF TRACHEOTOMY.
}

CASES WITH REMARKS.

BY

THOMAS SMITH, F.R.C.S.,

ASSISTANT-SURGEON TO ST. BARTHOLOMEW'S HOSPITAL AND TO THE HOSPITAI FOR GICK CHILDREN.

Received April 8th.-Read June 27th, 1865.

THe hindrances to the withdrawal of the canula and to the restoration of the passage of the larynx, to which I wish to draw attention, are such as only take effect after the performance of tracheotomy, and as a consequence of that operation. They have no reference to the persistence of any cause of obstruction that may have necessitated the introduction of the tracheal tube.

Case 1.-George S-, a delicate-looking, pallid boy, came into the Children's Hospital, April 4th, 1864. He had suffered from symptoms of laryngeal obstruction for some years, ever since falling into a canal and taking cold. 
During the day-time his breathing was difficult and stridulous, and occasionally at night he was seized with a violent paroxysm of dyspnœa ; which more than once put his life in great peril. At Dr. West's request, a fortnight after the child's admission I opened the trachea just below the cricoid cartilage. The operation gave immediate and permanent relief; but from the date of its performance the patient was never able either to wear a cork in the orifice of the canula, or to bear the removal of the tube for more than a few seconds.

The ability to inspire through the larynx was entirely lost, though on a momentary closure of the canula he could blow a whistle, and even speak in a harsh stridulous voice. As the child seemed to be gradually losing even this diminished expiratory power, about a fortnight after the operation he was put under chloroform (which was easily administered through the tracheal canula), and the finger being introduced into the throat, an examination was made. The epiglottis and aryteno-epiglottidean folds were found much thickened, indurated, and corrugated, so as satisfactorily to account for the dyspnœa under which he laboured previous to the performance of tracheotomy, but the condition was not sufficient to account for the complete inability to inspire from which he now suffered.

On examining the larynx from below, through the wound in the trachea, it was found that abundant vascular granulations had collected above and around the upper end of the canula, so as almost completely to block up the entrance to the larynx from below.

By the introduction of minute tents formed of the "Laminaria digitata," or sea-tangle weed, the cavity of the airtube above the canula was soon cleared of granulations, and its normal calibre restored; other means were also taken to remedy the chronic thickening about the upper part of the larynx. At the present date, however, the boy remains unrelieved; he still wears a canula in the trachea; his present state of health not justifying the adoption of further measures for his relief.

This case is related to show the rapidity with which, under 
some circumstances, granulations will collect around the tracheotomy tube, obstructing the passage of air through the larynx. I cannot but think, also, that it is worthy of remark that the ability to breathe through the larynx, which the child possessed in a limited degree, and with occasional interruptions, up to the time of the operation, was at once and entirely lost by the introduction of the tracheotomy tube; for from the date of the operation he was unable to inspire at all through the natural channel ; though, as usually happens, the expiratory power was not altogether lost. 'This phenomenon may frequently he observed in those who have had tracheotomy performed for laryngeal obstruction, namely, that what little power of inspiration through the larynx the patient possessed before the operation, is at once, for a time at least, lost, by the introduction of the tube into the trachea.

Case 2.-Fanny S-, æt. 4, was admitted into the Children's Hospital, November 15th, 1863, under Dr. West's care. She was suffering from diphtheria, having the characteristic exudation about the fauces, with albuminuria. Tracheotomy was performed four days after her admission, by Mr. Marsh, the house surgeon, the child at the time being apparently moribund. The operation was thoroughly successful; four days after its performance the tracheotomy tube was removed; the canula having been previously closed for some hours to ascertain that the larynx was permeable. Within a minute or two of the withdrawal of the tube the rima glottidis seemed suddenly to close, and the child ceased to breathe until the canula was replaced.

From time to time, during some weeks, the same experiment was tried, and always with the same result. In the spring of the following year the child went to a convalescent institution, still wearing the canula.

After her return to London, in April, 1864, Dr. West asked me to see her.

It was now more than four months since the operation; and I could not discover, after repeated examinations, any com- 
munication between the cavity of the larynx and the wound in the trachea. A solid mass of granulations seemed to have collected around the upper part of the tracheotomy tube, which completely prevented the passage of air. The child could not bear the canula closed for more than a few seconds, and if it were removed from the wound, the latter quickly began to contract, the edges inverting with each inspiration, so that in a minute or two it was difficult to replace the canula.

Under chloroform, which was administered through the wound, I succeeded in forcing a probe from below through the granulations into the cavity of the larynx ; this was followed by a small elastic catheter, which was tied to the canula. Three days afterwards a very small tent of the seatangle weed was passed from below, and was followed in a few days' time by a larger tent of the same material. This plan was pursued until there was a free communication between the larynx and trachea of sufficient size for respiratory purposes.

The child could now bear the canula closed during the whole day, while during the night it was left open; under chloroform the finger could be introduced through the upper opening of the larynx so as to meet a director or bougie passed from below through the wound in the trachea; there appeared to be no obstruction whatever to the passage of air, and by this time also the child had so far recovered her voice as to be able to speak in a loud harsh tone when the canula was closed.

Under these circumstances the tube was frequently withdrawn from the trachea, but it always had to be replaced within a few minutes, on account of impending asphyxia. At the very first inspiration after each withdrawal, the edges of the wound began to invert, and in the course of three or four minutes the tube was replaced with difficulty. Thinking that perhaps the collapse of the wound in the soft parts over the trachea might narrow the calibre of that tube and thus account for the difficulty of breathing without the canula, a gutta-percha plug was made to fit the orifice of the wound and prevent its contraction when the canula was withdrawn. 
On the only occasion upon which this plug was applied, symptoms of asphyxia came on instantly, the child being unable to make a single inspiration with it in the wound. For a month or two all treatment was abandoned as the child caught the measles.

September 20th, 1864.-Ten months after the operation the child was again examined; the opening between the trachea and larynx was found just large enough to admit a probe with difficulty; the voice was reduced to the smallest whisper, and the child could not bear the canula closed. By means of seaweed tents introduced through the wound, the communication between the trachea and larynx was again dilated to a fair size, so as to admit of the canula being kept constantly plugged during the day-time. As had occurred before, all attempts to keep the tube closed at night were ineffectual ; so soon as the child fell asleep, if the canula were closed, a violent attack of dyspnoea would come on, and, if sufficiently conscious, she would herself withdraw the plug.

In November, eleven months after the operation, the patient was in much the same condition, except that under the application of powdered alum to the lower part of the larynx the voice had improved remarkably, both in power and tone. In order to free the mucous membrane as much as possible from irritation, the external canula tube was cut short, so as only just to enter the trachea, and this, with a cork in its orifice, was worn during the day, while at night-time the cork was replaced by an internal cauula tube of the ordinary length.

By closing the orifice of the external tube with a piece of thin glass, so as to prevent the escape of air through it, one could examine with the ophthalmoscope the fissure leading from the trachea to the larynx. From the shape of this opening and the position assumed by its borders during speaking and inspiration, $I$ felt convinced it was the under aspect of the rima glottidis, and that the parts were quite free from granulations. There could now be no obstacle to the passage of air through the larynx, except such as might be offered by the muscles of the glottis. I therefore came to the conclusion 
that the difficulty of breathing through the larynx arose from a paralysis, or some imperfection of action either in time or power, of the dilator muscles, the crico-arytenoidei postici, and that the sudden symptoms of asphyxia which were wont to come on during sleep were due to a spasm of those muscles which close the glottis; $i$. e., arytenoid and crico-arytenoidei laterales. In fact, the whole muscular apparatus of the larynx seemed thrown out of working gear.

It is worthy of remark that though the child could breathe freely with the canula closed, yet she could not bear its entire removal for more than a minute or two without suffering greatly from dyspnœa which soon became insupportable. And again, that the tube could be kept closed at all times except during sleep.

I venture to suggest the following explanation of these circumstances-that the mental agitation caused by the dread of impending suffocation which the removal of the canula produced, was sufficient to derange the normal action of the laryngeal muscles, so as to prevent the regular dilation of the rima glottidis at each inspiratory act.

It might be also that the influence of the will was necessary to regulate and secure the due action of these muscles, the perfection of whose movements had been impaired; and that on this account inspiration through the larynx during sleep was impossible.

The threefold office of the larynx-as the organ of the voice, as a conduit for air, and as the portal of the respiratory cavity-necessitates in its intrinsic muscles various and complex functions. They have to act both as vocalizers and as the janitors of the passage to the lungs, in the latter capacity either maintaining the patency of the canal, or more exceptionally excluding foreign bodies or noxious vapours by closing the rima-glottidis.

It would seem that the operation of tracheotomy in a manner mars the perfection of the coincidence between the action of the laryngeal muscles and those of inspiration; that it is liable to cause undue irritability and disorderly action of the muscles of the glottis, and to interrupt their 
usual rhythm. Perhaps this is the effect of the enforced cessation of all exercise of function in the larynx which the operation entails; or, less likely, it is a reflex effect of the irritation caused by the canula.

These views are, I think, supported by the sequel of the case above quoted, as well as by others to which reference will be made hereafter.

When thirteen months had elapsed after the performance of the operation on Fanny S-, Dr. West kindly drew my attention to the account of a similar case by Dr. Sendler, ${ }^{1}$ of which the following is an abstract:

"A child aged one year and nine months had tracheotomy performed on the sixth day of membranous croup; the operation gave immediate and permanent relief, but was followed by an inability of the larynx to resume its functions. The first attempt to remove the tube was made on the fifth day after the operation: the larynx being free from any permanent obstruction, the attempt was unsuccessful as were several subsequent ones. Before long, the child could bear the canula plugged at all times, except during sleep, but still it was impossible entirely to remove it, as on each occasion dyspnce came on, increasing in severity until the tube was replaced. In the last attempt the child nearly lost its life, thereupon Dr. Sendler consulted Trousseau on the case, who replied as follows :

" ' I have had two infants who have retained the canula, the one for three months, the other for seven months; in both cases I believed that a chronic inflammation of the larynx had followed the croup, and twice also, as it has been pointed out by M. Passavant, I was only able to remove the canula on the forty-second and fifty-third day of the disease; whichlatter seemed to remain in a subacuteform for along time.

" At the time I observed these facts, my attention had not as yet been drawn to the partial or general paralysis which follows diphtheria, and now I am tempted to attribute (as you do) the extreme difficulty of inspiration to the para-

1 'Prager Vierteljahr.,' vol. sxxiv, p. 57. 
lysis of the posterior arytenoid muscles. Nevertheless, when this paralysis exists, it causes a difficulty in deglutition, which you do not mention-this difficulty causes the entrance of fluids into the larynx during swallowing, and here is one of the most formidable accidents which follow tracheotomy.

" ' Now if your patient drinks fluids easily, one must believe in the existence of a chronic inflammation of the aryteno-epiglottidean folds. Whatever it be, I am persuaded that the child will recover, and I advise you, whenever you can, to leave a closed canula in the trachea until the child has breathed for ten days in a natural manner. The canula with a dorsal opening is a useless and often dangerous instrument. For if you compare the calibre of the canula with that of the trachea, you will see that the section of the trachea is more than double the area of that of the canula; the air, therefore, can pass freely between them. On the other hand, the tissues project through the dorsal opening of the canula, and thereby the inflammation is kept up. Be therefore hopeful, and be sure that unless there be necrosis of the cartilages of the larynx (which is but little probable), the child will recover." "

After the receipt of Dr. Trousseau's reply, Dr. Sendler allowed the canula to remain three weeks without any attempts to remove it until the child could bear it closed throughout the day, and for an hour or two at night-time. The canula was now again entirely withdrawn; but during sleep, the first night after its removal, such urgent dyspnœa came on that it was replaced. After the lapse of a month three unsuccessful attempts were made at short intervals to withdraw the canula. During the next six weeks no attempt was made to remove it, but its orifice was kept almost constantly closed, for a short time even during sleep, and the size of the instrument was gradually diminished until it was finally removed on March 11th, four months after the performance of tracheotomy. On this occasion Dr. Sendler determined to reintroduce the tube only under the most pressing emergency.

Throughout the day on which the canula was removed, no 
difficulty in breathing was experienced, but at night-time soon after the child fell asleep, dyspnœa came on, increasing in severity until 11 p.m., when it attained its maximum of intensity, and the child's life seems to have been in some danger. From this time, however, an occasional pause in the severity of the symptoms took place, and by the mornin all cause for immediate anxiety had passed away, and the child slept quietly. On the second day after the removal of the canula the wound had closed, and the child suffered no further inconvenience.

Acting on Dr. Sendler's experience in this case, on January 13th, 1865, fourteen months after the operation, I removed the tube from Fanny S-'s trachea, and covered the wound with a piece of plaister. This was done in the morning, about 9.30 a.m., and the child was placed for the whole day under constant observation, lest sudden need should arise for the introduction of the canula. Great care was taken to prevent any hurried breathing, and she was not allowed to play with the other children, or even to speak, as the increased demand on her respiratory powers seemed to threaten a laryngeal spasm.

She passed a tranquil day, the wound by evening had contracted to less than half its ordinary dimensions. During sleep that night she had a slight attack of dyspnœa, which soon passed off; next day the wound had nearly closed, and she could speak very fairly, though the larynx seemed not sufficiently free to allow of continuous conversation. The passage for air seemed also too small to allow of any muscular exercise, as the necessity for more rapid breathing caused the child distress. After running upstairs, the respiration was hurried, noisy, and difficult; but her respiratory power seemed quite sufficient so long as she was free from any unusual excitement; she could speak well. By the 17th of January the wound was quite closed, and the breathing was not distressed; on the evening of this day the eruption of varicella began to appear on her body, and towards night difficulty of breathing came on; it increased in urgency throughout the night, until Mr. Marsh, the house-surgeon (whom I had 
advised to avoid the reintroduction of the tube if possible), found it necessary to give chloroform, having in view the strong probability that the obstruction to breathing was partly of a spasmodic nature.

The anæsthetic relieved the dyspnœa in a degree, but after a while the latter became more urgent, and at about 4 a.m. on the 18th, six leeches were applied over the larynx ; the bites bled freely and gave much relief. Towards morning, the dyspnœe gradually subsided, and when I saw her at 11 a.m. it had in a great measure passed away ; though the child was prostrate from loss of blood, anxiety, and fatigue. On this occasion I believe that the preservation of the child's life without the performance of tracheotomy, was entirely due to Mr. Marsh's active and judicious treatment.

January 18th.-Towards evening on this day, the dyspnœa again returned, and by midnight the child was in extreme danger from suffocation. The house-surgeon remained with her during the whole night, ready to reopen the trachea at a moment's notice. Towards the morning of the 19th the difficulty in breathing began to subside, and during the whole of that day a gradual improvement took place in the general as well as in the local symptoms. The night of the 20th she passed through with but little dyspncea, and on January 21st, eight days after the removal of the tube, her breathing was quite easy, and her voice better than it had ever been before. A few days after this she was discharged well.

The following case furnishes another striking example of a permanent impairment of function in the laryngeal muscles after tracheotomy.

I am indebted to Dr. Russell Reynolds for permission to publish this case, and to Dr. S. Ringer for the notes of the same.

Case 3.-Arthur $\mathrm{H}$ - was admitted into University College Hospital under Dr. Reynolds, December 13th, 1863. The child was suffering from diphtheria, and the same evening tracheotomy was performed by Dr. Ringer; this gave speedy relief to the symptoms of asphyxia, and was soon followed 
by a general improvement in the child's constitutional condition. The case subsequently progressed favorably, and on December 27th, a fortnight after tracheotomy, the tube was removed; it was, however, at once replaced, as without it the child could not breathe. After this it was frequently removed, and sometimes (especially latterly), it could be kept out for an hour or two at a time; but for longer than this it could not be dispensed with.

From time to time the child suffered from severe and even dangerous attacks of dyspnœa from the blocking up of the tube. Under a recurrence of these paroxysms his health began to fail, and in April of the following year it became evident that the dorsal part of the spine was carious, there being extreme angular curvature. Shortly after this the child died of bronchitis.

On post-mortem examination, two small ulcers were found on the mucous membrane of the trachea, just above the incision; these very slightly narrowed the calibre of the airtube; the vocal cords and cavity of the larynx were found perfectly healthy. "The slight contraction of the trachea," says Dr. Ringer, "was insufficient to cause any difficulty in breathing." No paralysis of any part of the body could be detected at any period of the child's illness.

I venture to include this case in the same category as that of Fanny S-, attributing the inability to breathe through the larynx to a loss or perversion of function in the muscles of the glottis, and the result of the post-mortem examination quite confirms this view of the case.

CASE 4.1-Dr. George Buchanan, of Glasgow, on March 23rd, 1864, performed tracheotomy on a child, aged three years, who had been suffering for some days with symptoms of diphtheria. The operation was perfectly successful, and the child made so rapid a recovery that on March 27th, four days after the operation, the tube was removed, and he was left breathing quietly and freely, partly through the larynx

1 'Brit. Med. Journal,' Sept. 7th, 1864. 
and partly through the wound. Next morning he was quite well, being lively and playful, and taking his food with an appetite. At midday, whilst running about the room, he suddenly felt a choking sensation, became very pale, and died in a very short time, just after his medical attendant arrived. The wound seemed free from obstruction; the exudation had disappeared from the fauces altogether-the cause of death was not ascertained.

It seems highly probable that death in this case occurred from a spasmodic closure of the rima glottidis. The result suggests the propriety of a tentative and cautious removal of the canula before its final withdrawal, lest, after the subsidence of the original cause of obstruction, there remain a dangerous irritability of the laryngeal muscles.

Chassaignac in his 'Leçons sur la Trachéotomie,' p. 84, recommends that after the operation the canula should never be removed until three days have elapsed, and that when it is desirable to withdraw the canula, it should be done by degrees, and should be kept out of the wound for gradually increasing intervals of time.

In Cases 2 and $3 \mathrm{I}$ have attributed the inability to inspire through the larynx to some imperfection in the action of its muscles, for the following reasons :

The larynx in each case was proved to be pervious to air by the ability to speak on the temporary closure of the orifice of the canula, and the patients acquired the power of breathing through the larynx for long intervals of time ; in fact, at all times except during sleep, provided that the canula was in the wound. On the removal of the canula, the power of breathing through the larynx, though continuing for a time, was suddenly and almost completely lost; while in one case the larynx was ascertained to be free from any permanent obstruction by post-mortem investigation; in another by tactile and visual examination. The imperfections of action in the laryngeal muscles, to which allusion is made, include losses of rhythm, losses of power, or an undue excitability and proneness to spasmodic contraction.

As has been before remarked, it is possible that the ope- 
ration of tracheotomy could produce such results in two ways, either by the sudden cessation of all movements in these muscles which it enforces, thereby interrupting the regular rhythmal action of the dilators of the glottis ; or by a reflex irritation or paralysis of the same muscles from the continued contact of the canula with the mucous membrane of the trachea.

The paralysis of the muscles of the extremities as a sequel of diphtheria is not of infrequent occurrence. Muscular anæsthesia and paralysis of the velum palati; disorderly action of the pharyngeal constrictors; loss of vocalizing power in the muscles of the larynx ; any or all of these may follow diphtheria. It might not, therefore, seem unreasonable to attribute to the same cause the disordered muscular action which occurred in the cases related above.

But supposing this view to be the true one, it is remarkable that the laryngeal muscles should be the only muscles affected, and that none of the cases above related, nor indeed any that have occurred in the Children's Hospital have suffered from any other form or degree of diphtheritic paralysis within the memory of the present medical staff.

Again, as opposed to this view, is the fact that the same temporary loss of function in the muscles of the larynx is liable to occur when tracheotomy has been performed for acute laryngitis, and that immediately after the performance of tracheotomy there ensues in almost all cases, for a time at least, a complete inability to inspire through the larynx.

In the following case the operation was performed for laryngitis :

J. J-, æt. 24, was admitted into St. Bartholomew's under Dr. Jeaffreson's care, January 4th, 1865. He had been suffering with symptoms of laryngitis one week. On the day of his admission the house-surgeon, Mr. Pearless, opened the trachea, and gave complete relief to all the most urgent symptoms. Fourteen days afterwards the canula was coughed out, and was at once replaced.

I saw him February 7 th, one month after the operation; 
he had for the last two days been wearing occasionally an instrument for directing the expired air through the larynx ; no attempt had been made to remove the canula, which latter he could not bear closed for more than a minute at a time. If its orifice were closed after drawing about fifteen or twenty inspirations through the larynx, with some difficulty and great anxiety, the glottis appeared to close suddenly and completely, and the air had at once to be readmitted through the wound. With the above-mentioned instrument in the canula his voice was fairly good in all but tone.

A laryngoscopic examination of the upper part of the larynx showed the mucous membrane to be highly congested and somewhat swollen. In the only view I could get of the vocal cords, the metallic lustre of the tracheotomy tube was so distractingly evident that I could not speak with any certainty of their condition. A few days after this he was attacked with fever, and was not again examined until March 27th, when convalescent.

March 27th, three months after the operation, he was exactly as when examined before his attack of fever. There was a fair power of voice, but no use of his larynx as an airpassage to the lungs. To free the mucous membrane from irritation, the long tube that he had worn since the operation was removed, and a much smaller and shorter one was introduced, so as only just to enter the trachea. Two days after this he bore the canula closed for five minutes, and on April the 3rd for nearly ten minutes; but on each occasion the attempt to keep in the plug was terminated by a sudden and complete closure of the glottis.

On April 3rd a view of the interior of the larynx was obtained; the aryteno-epiglottidean folds were slightly reddened and a little puffy. The vocal cords looked natural, and although from the difficulty of examining the patient and from the cloudy state of the sky, I obtained only one clear view of the rima glottidis, yet there seemed to be a free passage for air.

On examining the wound the communication between the larynx and trachea, though somewhat reduced in size, ap- 
peared quite sufficient for the purposes of respiration. The result of this examination, together with the sudden and choking kind of dyspnœa which attacked the patient on each occasion the canula was closed, make me believe that the difficulty of inspiration is due to an exalted irritability of the muscles of the glottis, and not to any permanent obstruction of the canal.

The rapid and abundant growth of granulations about the tracheotomy tube in the cases of George and Fanny Sfurnishes an additional reason, if any were needed, for early and repeated attempts to withdraw the canula so soon as the original cause of obstruction is at an end, though on this point so high an authority as $M$. Chassaigne remarks that " there may be inconvenience attached to a too early removal of the canula, and there can be no harm in leaving it in."1 2

The following case, related by Dr. Steiner, furnishes an example of the entire obliteration of the canal of the larynx after tracheotomy :

CASE.-W. A-, æt. 2, a rickety child, had tracheotomy performed for croup July, 1862. The child recovered from the symptoms of croup, but every attempt to withdraw the canula from the trachea was immediately followed by symptoms of impending suffocation, and this condition of things continued to the time of his death, nine months after the operation, of hydrocephalus. On examination it was found that the opening of the larynx had entirely disappeared, the canal being perfectly obliterated by a firm and almost cartilaginous cicatrix mass which, of course, could have been produced only by deep ulceration following on the croupal exudation.

From the account of this case, as published in the foregoing report, ${ }^{3}$ one would almost feel in doubt whether ad-

1 'Leçons sur la Tracheotomie,' p. 84.

2 The retention of the canula in the wound may produce ulceration of the mucous membrane, or necrosis of the cricoid cartilage or tracheal rings.

' This case is published in the 'Prag. Vierteljahrschrift.' The account of the post-mortem was furnished me by Dr. West, who received it from Dr. Steiner.

VoL. XLVIII. 
hesion had taken place between the opposed surfaces of the vocal cords rendered bare by ulceration, as being the only spot in the larynx where adhesion could take place between its opposite walls; or whether, as nearly happened in the cases of Fanny and George S-, the canal of the larynx had become obliterated by a mass of organized granulations.

From the consideration of the foregoing cases it appears that among the obstacles to the re-establishment of natural respiration after tracheotomy are the following:

1st. A narrowing or complete obliteration of the passage of the larynx by the growth of granulations above and around the canula.

2nd. An impairment or complete loss of those functions of the muscles of the larynx which regulate the admission or exclusion of air through the rima glottidis.

3rd. Adhesion of the opposed surfaces of the vocal cords.

Having in view the avoidance of the contingencies above enumerated, I would draw attention to an instrument invented by Luer, of Paris, to be worn in the orifice of the canula as soon as practicable after the performance of tracheotomy.

This instrument, by means of a bullet-valve, ensures a free entrance of air by the wound through the canula, while it obliges all air leaving the cavity of the chest to pass out through the larynx.

The advantages which I think we may reasonably expect from such an instrument are the following :

1st. Its occasional and early employment favours the expulsion of false membrane and tenacious mucus from the cavity of the larynx during coughing.

2nd. Later in the case it may serve as an effectual and safe means of ascertaining the permeability of the larynx to the passage of air.

3rd. It restores the power of speech, and enables the laryngeal muscles to exercise at least some part of their ordinary functions, so as to prevent the injury they would suffer by complete disuse.

4th. By accustoming the larynx to the transit of air through 
its cavity, it may diminish the irritability of the intrinsic muscles.

M. Luer's instrument (specimens of which I beg to place before the Society) quite fulfils the most important ends; while its costliness, weight, the want of simplicity in the manner of its attachment to the canula, and the fact that it requires a special canula for its adaptation, have interfered with its extensive employment.

I have therefore employed a more simple means for attaining the same end. This little appliance (specimens of which I beg to place before the Society) is light and inexpensive, and can be adjusted to any canula of corresponding size.

For the bullet valve of Luer is substituted an india-rubber flap, and its attachment to the canula is secured by a plugshaped extremity. It is made by Messrs. Fergusson, of Giltspur Street, and it will be found to answer all the purposes of Luer's instrument. 